\title{
Effect of Glucose Control on Delayed Gastric Emptying in Diabetic Patients: Author's Reply
}

TO THE EDITOR: We thank Dr. Kim for her interest in our paper. ${ }^{1}$ We agree regarding the issues raised in the correspondence to the editor. The study under discussion was a retrospective analysis of an available serum hemoglobin A1C (HbA1C) level near the performance of a gastric emptying scan. As a result of this limitation, assessment of preprandial or postprandial glucose values as well as duration of diabetes and presence of diabetic complications could not be performed. ${ }^{2-5}$

Categorical analysis of our study patients with abnormal $\mathrm{HbA1C}$ (defined as greater than or equal to $7 \%$ ) and abnormal gastric emptying times (defined as greater than or equal to $120 \mathrm{mi}$ nutes) showed no statistically significant association $(P=0.525)$. Because analyses using gastric emptying time and $\mathrm{HbA} 1 \mathrm{C}$ as continuous variables did not show conclusive differences, categorical analysis was not performed using cut-off times of 90 or 180 minutes. Further studies prospectively evaluating pre and postprandial glycemia, prolonged gylcemic control measured by multiple serum $\mathrm{HbA} 1 \mathrm{C}$ levels over time, age and duration of diabetes diagnosis as well as presence of diabetic complications can further elucidate the relationship of these variables with one another and their effects on gastric emptying time.

\author{
Carlos Palacio ${ }^{1}$ and Kenneth J Vega ${ }^{2}$ \\ ${ }^{1}$ Department of Medicine \\ University of Florida College of Medicine-Jacksonville \\ Jacksonville, Florida, USA \\ ${ }^{2}$ Division of Digestive Diseases and Nutrition \\ University of Oklahoma Health Sciences Center \\ Oklahoma City, Oklahoma, USA
}

1. Reddy S, Ramsubeik K, Vega KJ, Federico J, Palacio C. Do HbA1C levels correlate with delayed gastric emptying in diabetic patients? J Neurogastroenterol Motil 2010;16:414-417.

2. Camilleri M. Clinical practice. Diabetic gastroparesis. N Engl J Med 2007;356:820-829.

3. Kuo P, Rayner CK, Jones KL, Horowitz M. Pathophysiology and management of diabetic gastropathy: a guide for endocrinologists. Drugs 2007;67:1671-1687.

4. Rayner CK, Horowitz M. Gastrointestinal motility and glycemic control in diabetes: the chicken and the egg revisited? J Clin Invest 2006;116:299-302.

5. Shiraiwa T, Kaneto H, Miyatsuka T, et al. Post-prandial hyperglycemia is an important predictor of the incidence of diabetic microangiopathy in Japanese type 2 diabetic patients. Biochem Biophys Res Commun 2005;336:339-345.

\section{Conflicts of interest: None.}

